\title{
Inf-Sup Control of Discontinuous Piecewise Affine Systems
}

\author{
J. Spjøtvold ${ }^{1, *}$, E. C. Kerrigan ${ }^{2}$, S. V. Raković ${ }^{3}$, D. Q. Mayne ${ }^{4}$ and T. A. Johansen ${ }^{1}$ \\ ${ }^{1}$ Department of Engineering Cybernetics, Norwegian University of Science and Technology, N-7491 Trondheim, Norway. \\ 2 Department of Aeronautics and Department of Electrical and Electronic Engineering, Imperial College London, London \\ SW7 2AZ, United Kingdom \\ ${ }^{3}$ Automatic Control Laboratory, Swiss Federal Institute of Technology, Physikstrasse 3, CH-8092 Zurich, Switzerland \\ ${ }^{4}$ Department of Electrical and Electronic Engineering, Imperial College London, London SW7 2AZ, United Kingdom
}

\begin{abstract}
SUMMARY
This paper considers the worst-case optimal control of discontinuous piecewise affine (PWA) systems, which are subject to constraints and disturbances. We seek to pre-compute, via dynamic programming, an explicit control law for these systems when a piecewise affine cost function is utilized. One difficulty with this problem class is that, even for initial states for which the value function of the optimal control problem is finite, there might not exist a control law that attains the infimum. Hence, we propose a method that is guaranteed to obtain a sub-optimal solution, and where the degree of sub-optimality can be specified a priori. This is achieved by approximating the underlying sub-problems with a parametric piecewise linear program. Copyright (c) 2007 John Wiley \& Sons,
\end{abstract} Ltd.

KEY WORDS: Optimal control. Piecewise affine systems. Parametric programming. Approximate solutions to inf-sup problems.

\section{Introduction}

Gal and Nedoma proposed a complete algorithm for solving parametric linear programs (pLP) as early as [1] and in the following years the topic was subject of substantial research, see e.g. [2,3] and references therein. Parametric programming has had a resurgence of interest recently due to the observation that explicit control laws for some model predictive control problems [4] can be obtained by viewing the initial state as a vector of parameters [5-7]. As researchers tried to characterize the solution to more difficult optimal control problems (piecewise affine systems, uncertain systems, nonlinear systems etc.), several variations of the dynamic programming approach originally proposed by Bellman [8] has been employed [9-19]. The dynamic programming approach for inf-sup (or more commonly min-max) control was introduced for linear systems as early as [9] and in a more general framework in [10]. The methods $[9,10]$ have served as the foundation for a large number of publications (see e.g. [12-19]) and will also be utilized in this paper.

Recently, methods for computing explicit control laws for discrete-time piecewise affine (PWA) systems with constraints have been reported in the control literature [12, 15, 16, 20-23]. There are

${ }^{*}$ Correspondence to: jorgesp@itk.ntnu.no 
several reasons why obtaining explicit solutions to this problem class has been of interest; $i$ ) Piecewise affine systems arise both naturally (e.g. backlash [24] and hysteresis) and as approximations to nonlinear systems. Piecewise affine models are also equivalent to a large class of hybrid systems [25]. ii) An explicit solution to an optimal control problem offers several advantages compared to the on-line counterpart. The required on-line computation time is reduced, rendering optimal control applicable also for fast systems. In addition, the explicit solution makes it possible to off-line verify the correctness of the control law, which is a key point in safety critical applications. iii) Although several authors have addressed the topic of obtaining explicit solutions to optimal control problems for non-linear systems [26-29], these methods are often computationally demanding, sub-optimal and may lack stability guarantees. Even for linear systems with quadratic cost functions it is difficult to incorporate disturbances/uncertainties as the min-max formulation results in a non-convex problem that is not easily divided into sub-problems. $i v$ ) Recent results on parametric linear programming [30-33] and the evaluation of piecewise affine control laws [34-36] are directly applicable to optimal control of discrete-time PWA systems with linear cost functions as these problems can be solved explicitly with a series of pLPs.

In $[18,19]$ a relaxed dynamic programming procedure is proposed. The procedure reduces the computational complexity by relaxing the demands for optimality. The existence of a solution is assumed in $[18,19]$, which is different from the objective in this paper; to obtain a sub-optimal solution only when the minimum does not exist.

In [16] a dynamic programming approach for obtaining explicit control laws to continuous PWA systems not affected by disturbances is presented. Discontinuous PWA systems are briefly mentioned, but the topic is not treated in detail; for instance that an optimizer exists ${ }^{\dagger}$ cannot be guaranteed. In addition, the authors represent the domain of the PWA state update equation by closed polyhedra, as described in [37], and therefore small gaps are introduced in the domain of the state update equation. Consequently, from a theoretical point of view, the state trajectory may vanish. On the other hand, one can argue that the control scheme is to be implemented on a microchip or computer and therefore is subject to a finite arithmetic precision. In this paper we will look at the problem from a theoretical viewpoint and remove the need to introduce small gaps.

An alternative way of describing a discontinuous PWA system is to transform the state update equation into a difference inclusion by performing a regularization, see e.g. [38]. With this system description, the successor state may be set-valued for a given initial state, control input and disturbance. We seek to avoid this situation by still treating the system as a difference equation.

In [15] continuous PWA systems with piecewise affine cost functions subject to state- and inputdependent disturbances are considered. This paper presents a non-trivial extension of this approach to discontinuous PWA systems. Methods for computing explicit control laws for this problem class have not yet been reported in the literature, hence, in this paper we outline the foundation for a complete computational procedure.

In this paper we represent the domain of PWA systems by a union of a finite number of open, closed and/or neither open nor closed polyhedra. A solution to the optimal control problem may not exist in this case. However, solutions for which the cost is arbitrarily close to the infimum/supremum are guaranteed to exist. We propose a procedure that obtains a sub-optimal solution to the optimal control problem when the solution does not exist, and the exact solution when it does. This approach does

\footnotetext{
${ }^{\dagger}$ Given an optimization problem, e.g. $J^{*}:=\inf _{x \in X} f(x)$, we say that an optimizer exists if the infimum is attained, i.e. $\exists x^{*} \in X$ such that $J^{*}=f\left(x^{*}\right)$. 
not introduce gaps in the domain of the state update equation, we do not assume that a solution to the optimal control problem exists, and the state update equation is not transformed into a difference inclusion, and thus, the dynamic programming approach is relatively simple from the theoretical point of view. In addition, the proposed procedure allows the degree of sub-optimality to be specified $a$ priori.

PAPER STRUCTURE: Section 2 introduces basic notation and presents an illustrative and motivating example. In Section 3 we introduce the basic building block in the paper, namely how one can obtain sub-optimal solutions to parametric linear programs with strict and non-strict inequality constraints. This building block is then used to obtain sub-optimal solutions to minimization of PWA functions in Section 4, to min-max problems in Section 5, and finally it is demonstrated in Section 6 how these procedures can be used in the dynamic programming approach for the purpose of obtaining explicit sub-optimal solutions to robust optimal control problems for discontinuous PWA systems.

\section{Preliminaries}

\subsection{Basic notation and fundamental results}

For completeness we recall some standard notation and definitions. The affine hull of a set $S$ is the intersection of all affine sets containing $S$, and is denoted $\operatorname{aff}(S)$. The dimension of a set $S \subseteq \mathbb{R}^{n}$ is the dimension of aff $(S)$, and is denoted $\operatorname{dim}(S)$; if $\operatorname{dim}(S)=n$, then $S$ is said to be full-dimensional. The closure of a set $S$ is denoted $\operatorname{cl}(S)$. The relative interior of a set $S$ is the interior relative to aff $(S)$, i.e.

$$
\operatorname{relint}(S):=\{x \in S \mid B(x, r) \cap \operatorname{aff}(S) \subseteq S \text { for some } r>0\},
$$

where the ball $B(x, r):=\{y \mid\|y-x\| \leq r\}$ and $\|\cdot\|$ is any norm. We denote the orthogonal projection of a set $S \subseteq \mathbb{R}^{n} \times \mathbb{R}^{m}$ to $\mathbb{R}^{n}$ by $\operatorname{Proj}_{x} S:=\left\{x \in \mathbb{R}^{n} \mid \exists u \in \mathbb{R}^{m}\right.$ such that $\left.(x, u) \in S\right\}$.

A polyhedron is the intersection of a finite set of open and/or closed halfspaces. Note that our definition of a polyhedron differs from the most common definition in the sense that we do not require the halfspaces to be closed. The reason for this variation is to be able to represent discontinuous piecewise affine functions. A union of a finite number of polyhedra will be abbreviated FUP. We will adopt a similar notation to that presented in [39] with regards to the concept of extended real valued functions. Thus, a function $f$ is allowed to take values in $\overline{\mathbb{R}}=[-\infty, \infty]$. Recall also that the infimum is the greatest lower bound of a set $S \subseteq \mathbb{R}$, defined as a quantity $m$ such that no member of the set is less than $m$, but if $\varepsilon$ is any positive quantity, however small, there is always one member $s$ that is less than $m+\varepsilon$. Moreover, we introduce the notation $\inf _{C} f:=\inf _{x \in C} f(x):=\inf \{f(x) \mid x \in C\}$ and $\sup _{C} f:=\sup _{x \in C} f(x):=\sup \{f(x) \mid x \in C\}$. By convention we have inf $\emptyset=\infty$ and $\sup _{\emptyset} f=-\infty$, and hence,

$$
\arg \min _{x \in C} f(x):= \begin{cases}\left\{x \in C \mid f(x)=\inf _{C} f\right\} & \text { if } \inf _{C} f \neq \infty \\ \emptyset & \text { if } \inf _{C} f=\infty\end{cases}
$$

and

$$
\arg \max _{x \in C} f(x):= \begin{cases}\left\{x \in C \mid f(x)=\sup _{C} f\right\} & \text { if } \sup _{C} f \neq-\infty \\ \emptyset & \text { if } \sup _{C} f=-\infty\end{cases}
$$

We say that the infimum (supremum) of $f$ over $C$ is attained (or the minimum (maximum) exists) if $\arg \min _{x \in C} f(x) \neq \emptyset\left(\arg \max _{x \in C} f(x) \neq \emptyset\right)$. For a function $f: \mathbb{R}^{n} \rightarrow \overline{\mathbb{R}}$, the domain of $f$ is 
defined as the set

$$
\operatorname{dom}(f):=\left\{x \in \mathbb{R}^{n} \mid-\infty<f(x)<\infty\right\} .
$$

Whenever we refer to a function $f$ or mapping $F$ having a certain property, we implicitly mean that the property holds only on the domain of $f$ or $F$, e.g. if we say that $f$ is continuous, it is continuous at every $x \in \operatorname{dom}(f)$.

A function $f: \mathbb{R}^{n} \rightarrow \mathbb{R}^{m}$ is piecewise affine (PWA) on its domain if dom $(f)$ is the union of finitely many polyhedra, relative to each of which $f(\cdot)$ is affine. If $X \subseteq \mathbb{R}^{n}$ and $Y \subseteq \mathbb{R}^{m}$, then $2^{Y}$ is the power set (set of all subsets) of $Y$ and a set-valued map is defined as $F: X \rightarrow 2^{Y}$. For notational simplicity, we use double arrows to specify that a mapping is set-valued, i.e. set-valued maps are specified as $F: X \rightrightarrows Y$. We say that the set-valued map $F: \mathbb{R}^{n} \rightrightarrows \mathbb{R}^{m}$ is piecewise polyhedral if the graph of $F$, defined as $\operatorname{gph}(F):=\{(x, u) \mid u \in F(x)\}$, is a FUP. A function $f: \mathbb{R}^{n} \rightarrow \mathbb{R}^{m}$ is a selection of the set-valued map $F: \mathbb{R}^{n} \rightrightarrows \mathbb{R}^{m}$ if $f(x) \in F(x)$ for all $x \in \operatorname{dom}(F)$. If $F: X \rightrightarrows Y$ is a mapping, then the restriction of $F$ to the domain $D$ is written $\left.F\right|_{D}: D \rightrightarrows Y$.

Throughout we will use the superscript * to distinguish between optimizers and decision variables, e.g. for the problem $\min _{x} f(x), x$ is the decision variable and $x^{*}$ denotes an optimizer. We also let such that be abbreviated s.t.

Given the optimization problem

$$
J^{*}:=\inf _{u \in U} f(u),
$$

we denote by $\varepsilon$ - $\arg \min _{u \in U} f(u)$ the set of values of $u \in U$ for which $f(u) \leq J^{*}+\varepsilon$, that is,

$$
\varepsilon-\arg \min _{u \in U} f(u):=\left\{u \in U \mid f(u) \leq J^{*}+\varepsilon\right\} .
$$

The following observation follows directly from the definition of the infimum of a set, but for completeness we include a proof.

Lemma 1. Assume that $J^{*}>-\infty$, that $U \subseteq \mathbb{R}^{n}$ is a non-empty set and $f: \mathbb{R}^{n} \rightarrow \mathbb{R}$, then $\varepsilon-\arg \min _{u \in U} f(u) \neq \emptyset$ for all $\varepsilon>0$.

\subsection{Example}

In this section we present a motivating example where we show that for a simple PWA function the minimum does not exist. The example also shows that to intuitive strategies to overcome this problem leads to other undesired effects, such as set-valued solutions and gaps in the domain of the solution. The reader may interpret $x$ as the initial state and $u$ as the control input in the following example to see that there may be a problem to compute a control law that attains the infimum. It should also be noted that minimization of PWA functions are sub-problems in the dynamic programming recursion that may be utilized to compute explicit solutions to optimal control problems.

\section{Example 1.}


Consider the following function:

$$
\begin{aligned}
& f(x, u):= \begin{cases}f_{1}(x, u):=2 & \text { if } \quad(x, u) \in P_{1} \\
f_{2}(x, u):=-u+x & \text { if } \quad(x, u) \in P_{2} \\
f_{3}(x, u):=-2 & \text { if } \quad(x, u) \in P_{3}\end{cases} \\
& P_{1}:=\{(x, u) \in \mathbb{R} \times \mathbb{R} \mid u \geq 0,-5 \leq x \leq 5\}, \\
& P_{2}:=\{(x, u) \in \mathbb{R} \times \mathbb{R} \mid u<0,-5 \leq x \leq 5\}, \\
& P_{3}:=\{(x, u) \in \mathbb{R} \times \mathbb{R} \mid 5<x \leq 7\},
\end{aligned}
$$

which is to be minimized with respect to $u$ for all values of $x \in \mathcal{X}:=[-5,7]$, that is,

$$
J^{*}(x):=\inf _{u} f(x, u), \quad \forall x \in \mathcal{X} .
$$

Figure 1(a) illustrates $f(0, u)$ for $u \in[-7,7]$; clearly the infimum $J^{*}(0)=0$ cannot be attained, that is, $\nexists u^{*}$ such that $f\left(0, u^{*}\right)=J^{*}(0)=0$. In fact, a minimum does not exist for any $x \in[-5,2)$. The value function $J^{*}(\cdot)$ is depicted in Figure 1(b) and it is indicated where a solution does not exist. Two strategies that might be natural to consider in order to overcome this problem is to either treat $f(\cdot)$ as set-valued, or to introduce small gaps in the domain of $f(\cdot)$, such that each affine function is defined over a closed polyhedron. If we treat $f(\cdot)$ as a set-valued map by removing the strict inequalities:

$$
\bar{f}(x, u):=\left\{f_{i}(x, u) \mid i \in \mathcal{M}(x, u)\right\},
$$

where $\mathcal{M}(x, u):=\left\{i \mid \exists i\right.$ s.t. $\left.(x, u) \in \mathrm{cl}\left(P_{i}\right)\right\}$, then the naive approach of choosing the minimum of $\bar{f}(\cdot)$ will change the value function compared to original the problem, see Figure 2(a). In addition, we see that even though $\bar{f}(x, \cdot)$ attains its minimum for all $x \in \mathcal{X}$, this is not case for $f(x, \cdot)$.

Introducing small gaps in the domain of the function, that is,

$$
\begin{aligned}
& \tilde{f}(x, u):=\left\{\begin{array}{lll}
2 & \text { if } \quad(x, u) \in \tilde{P}_{1} \\
-u+x & \text { if } \quad(x, u) \in \tilde{P}_{2} \\
-2 & \text { if } \quad(x, u) \in \tilde{P}_{3}
\end{array}\right. \\
& \tilde{P}_{1}:=\{(x, u) \in \mathbb{R} \times \mathbb{R} \mid u \geq 0,-5 \leq x \leq 5\}, \\
& \tilde{P}_{2}:=\{(x, u) \in \mathbb{R} \times \mathbb{R} \mid u \leq-\delta,-5 \leq x \leq 5\}, \\
& \tilde{P}_{3}:=\{(x, u) \in \mathbb{R} \times \mathbb{R} \mid 5+\delta \leq x \leq 7\},
\end{aligned}
$$

where $\delta>0$, will result in that $\tilde{f}(x, \cdot)$ is undefined for some $x \in \mathcal{X}$, see Figure 2(b).

We see from the above example that, even for a very simple PWA function, a minimum does not exist. In the sequel, we will propose a procedure that guarantees that for all $x$ for which the infimum is bounded, we obtain a $u^{*}$ such that $f\left(x, u^{*}\right) \leq J^{*}(x)+\varepsilon$, where the scalar $\varepsilon>0$ can be specified $a$ priori.

\section{3. $\varepsilon$-optimal solutions to parametric linear programs with strict and non-strict inequality constraints}

In this section we consider the problem of finding $\varepsilon$-optimal solutions to parametric linear programs with strict and non-strict inequalities. We propose a procedure that will be repeatedly used in 


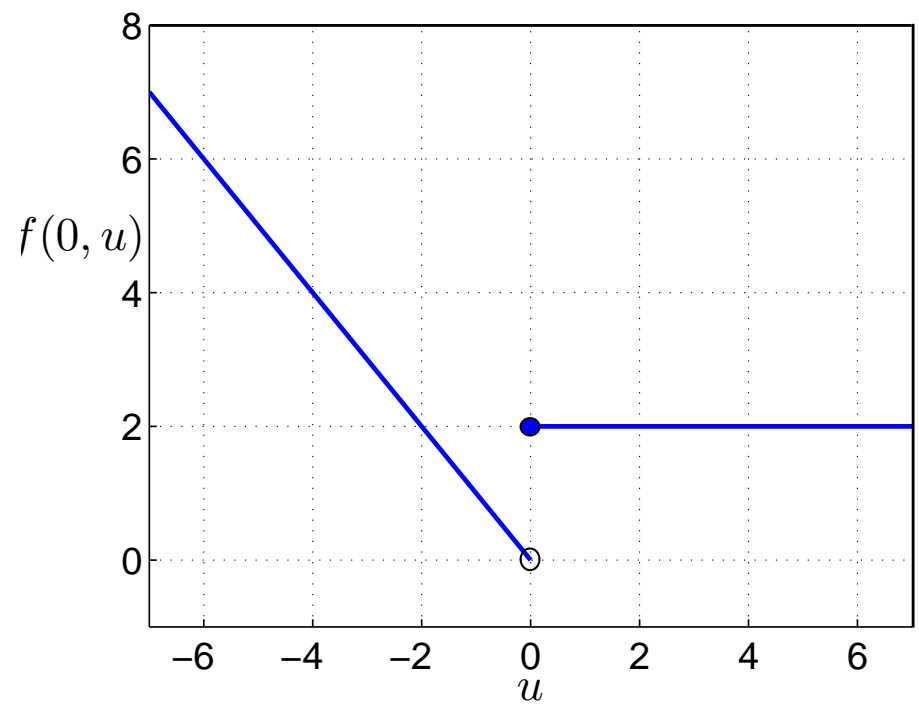

(a) Illustration of $f(0, \cdot)$. At the discontinuity the filled circle indicates the value of $f(0, \cdot)$, i.e. $f(0,0)=2$.

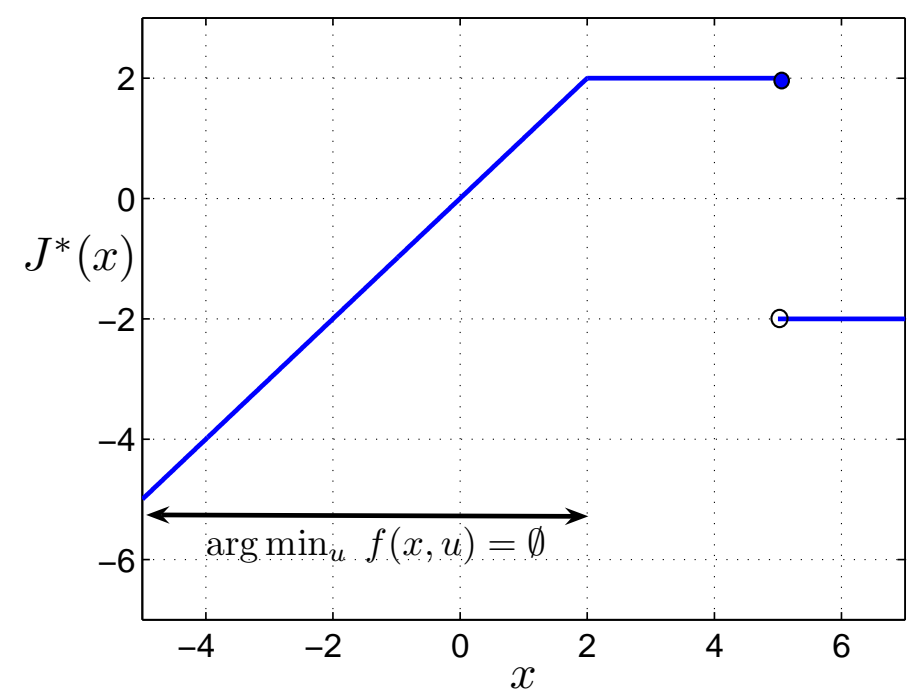

(b) Illustration of $J^{*}(\cdot)$. At the discontinuity the filled circle indicates the value of $J^{*}(\cdot)$ i.e. $J^{*}(5)=2$. For $x \in[-5,2)$ we have $\arg \min _{u} f(x, u)=\emptyset$.

Figure 1. Exact solutions for minimization of (1a).

subsequent sections for the purpose of obtaining sub-optimal solutions to optimal control problems with piecewise affine cost and constraints that are a finite union of polyhedra (FUP). 


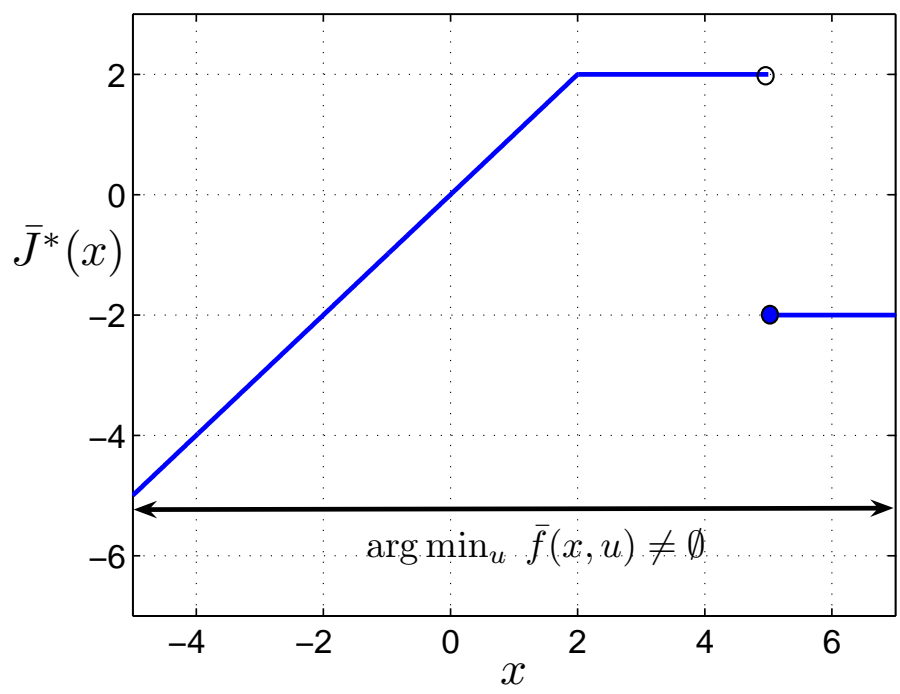

(a) Illustration of $\bar{J}^{*}(x):=\inf _{u} \bar{f}(x, u)$. It is concluded that $\exists u^{*}$ s.t $\bar{J}^{*}(x)=$ $\bar{f}\left(x, u^{*}\right)$ for all $x \in[-5,2)$, however, $\nexists u^{*}$ s.t $J^{*}(x)=f\left(x, u^{*}\right)$, cf. Figure 1(b). Moreover, $\bar{J}^{*}(5)=-2 \neq J^{*}(5)=2$.

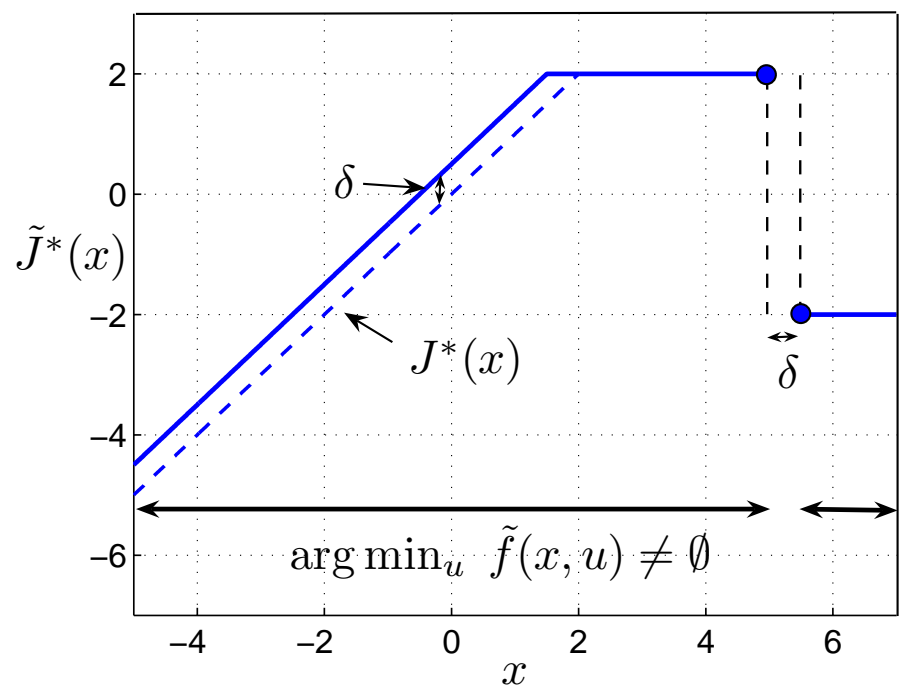

(b) Illustration of $\tilde{J}^{*}(x):=\inf _{u} \tilde{f}(x, u)$. The dotted line depicts $J^{*}(\cdot)$. The solid line depicts $\tilde{J}^{*}(\cdot)$. We see that in the interval $5<x<5+\delta$ the value function is undefined. In the interval $5+\delta \leq x \leq 7, \tilde{J}^{*}(\cdot)$ coincides with $J^{*}(\cdot)$.

Figure 2. Alternative approaches for the solution of (1a). 
Consider the problem

$$
\begin{aligned}
\mathbb{P}(x): \quad J^{*}(x) & :=\inf _{u}\left\{c^{T} u \mid(x, u) \in \mathcal{Z}\right\} \\
\mathcal{Z} & :=\left\{(x, u) \in \mathbb{R}^{n} \times \mathbb{R}^{m} \mid \begin{array}{l}
A u+B x \leq d \\
E u+F x<g
\end{array}\right\},
\end{aligned}
$$

which is to be solved for all $x \in \mathcal{X}$, where $\mathcal{X}$ is defined as:

$$
\mathcal{X}:=\left\{x \in \mathbb{R}^{n} \mid \exists u \text { s.t. }(x, u) \in \mathcal{Z}\right\} \cap\left\{x \in \mathbb{R}^{n} \mid J^{*}(x)>-\infty\right\}=\operatorname{dom}\left(J^{*}\right),
$$

and $c, A, B, d, E, F$ and $g$ are matrices with suitable dimensions.

The constraint set $\mathcal{Z}$ defines the set-valued map $U: \mathcal{X} \rightrightarrows \mathbb{R}^{m}$ given by

$$
U(x):=\{u \mid(x, u) \in \mathcal{Z}\},
$$

and hence (4) can be written as

$$
J^{*}(x)=\inf _{u}\left\{c^{T} u \mid u \in U(x)\right\} .
$$

We make the following standing assumption:

Assumption 1. For any parametric optimization problem that can be expressed as $J^{*}(x):=$ $\inf _{u}\left\{c^{T} u \mid(x, u) \in \mathcal{Z}\right\}$, we assume that $J^{*}(x)>-\infty$ for all $x$.

The above assumption is included to ensure that the infimum (or supremum if the problem is posed as $\left.J^{*}(x):=\sup _{u}\left\{c^{T} u \mid(x, u) \in \mathcal{Z}\right\}\right)$ is bounded for the pLPs posed throughout the paper. Note that the above assumption also has the immediate consequence that if $J^{*}(x) \neq \infty$ for some $x$, then $\mathcal{Z} \neq \emptyset$ and $\mathcal{X}$ is polyhedral.

For pLPs with only non-strict inequalities the following is well-known $[1,2,33,40]$ :

Theorem 1 (Solution properties for pLPs) Consider the $p L P$

$$
\hat{J}^{*}(x):=\min _{u}\left\{c^{T} u \mid(x, u) \in \operatorname{cl}(\mathcal{Z})\right\},
$$

which is to be solved for all values of $x \in \widehat{\mathcal{X}}$, where

$$
\begin{aligned}
\widehat{\mathcal{X}} & :=\left\{x \in \mathbb{R}^{n} \mid \exists u \text { s.t. }(x, u) \in \operatorname{cl}(\mathcal{Z})\right\} \cap\left\{x \in \mathbb{R}^{n} \mid \hat{J}^{*}(x)>-\infty\right\} \\
& =\operatorname{dom}\left(\hat{J}^{*}\right),
\end{aligned}
$$

and $\mathcal{Z}$ is defined in $(4 \mathrm{~b})$.

i) There exists a continuous and PWA function $u^{*}: \widehat{\mathcal{X}} \rightarrow \mathbb{R}^{m}$ that satisfies

$$
u^{*}(x) \in \arg \min _{u}\left\{c^{T} u \mid(x, u) \in \operatorname{cl}(\mathcal{Z})\right\}, \forall x \in \widehat{\mathcal{X}} .
$$

ii) The value function $\hat{J}^{*}: \widehat{\mathcal{X}} \rightarrow \mathbb{R}$ is continuous, convex and piecewise affine.

Remark 1. Note that according to the most common definitions of a continuous point to set map [4143], the mapping $x \mapsto \arg \min _{u}\left\{c^{T} u \mid(x, u) \in \mathrm{cl}(\mathcal{Z})\right\}$ is continuous, and several continuous selections are available; for instance minimum norm, Steiner point, or any extremal selector. For computational approaches, see [30,44,45] 
Before we present a theorem that provides a procedure for obtaining $\varepsilon$-optimal solutions for parametric linear programs with strict and non-strict inequalities, we recall a fundamental result for support functions to convex sets, formulated as a lemma for clarity of presentation [46, page 112]:

Lemma 2. If $S \subseteq \mathbb{R}^{m}$ is a convex set and $c \in \mathbb{R}^{m}$ is given, then

$$
\inf _{u}\left\{c^{T} u \mid u \in S\right\}=\inf _{u}\left\{c^{T} u \mid u \in \operatorname{cl}(S)\right\}=\inf _{u}\left\{c^{T} u \mid u \in \operatorname{relint}(S)\right\} .
$$

We cannot immediately use the above lemma in a parametric setting, so we provide the following corollary:

Corollary 1. Consider (4) and (7). Since $\mathcal{Z}$ is polyhedral we have that

$$
J^{*}(x)=\hat{J}^{*}(x) \text { for all } x \in \mathcal{X}=\operatorname{dom}\left(J^{*}\right) .
$$

Proof:

Using Lemma 2 we have

$$
\inf _{u}\left\{c^{T} u \mid u \in U(x)\right\}=\inf _{u}\left\{c^{T} u \mid u \in \operatorname{cl}(U(x))\right\}, \quad \forall x \in \operatorname{dom}\left(J^{*}\right) .
$$

We now need to show that

$$
\inf _{u}\left\{c^{T} u \mid u \in \operatorname{cl}(U(x))\right\}=\inf _{u}\left\{c^{T} u \mid(x, u) \in \operatorname{cl}(\mathcal{Z})\right\}, \quad \forall x \in \operatorname{dom}\left(J^{*}\right) .
$$

Defining $\bar{U}(x):=\{u \mid(x, u) \in \operatorname{cl}(\mathcal{Z})\}$ we can write (7) as

$$
\inf _{u}\left\{c^{T} u \mid u \in \bar{U}(x)\right\},
$$

and since $\mathcal{Z}$ is polyhedral it trivially follows that

$$
\operatorname{cl}(U(x))=\left\{\begin{array}{l|l}
u & \begin{array}{l}
A u \leq d-B x \\
E u \leq g-F x
\end{array}
\end{array}\right\}=\bar{U}(x) .
$$

A parametric optimization is said to be a parametric piecewise $L P$ if the set of parameters for which the infimum is bounded is the union of a finite number of polyhedra, relative to each of which the problem reduces to a pLP. Consider $\mathbb{P}(\cdot)$ and define the parametric piecewise LP $\left(\hat{J}^{*}(\cdot)\right.$ is PWA and equal to $J^{*}(\cdot)$ on $\mathcal{X}$ ):

$$
\begin{aligned}
\mathbb{P}_{\varepsilon}(x): V_{\varepsilon}^{*}(x) & :=\min _{(u, t)}\left\{t \mid(x, u, t) \in \mathcal{Z}_{\varepsilon}\right\}, \\
\mathcal{Z}_{\varepsilon} & :=\left\{\begin{array}{l|l}
(x, u, t) \in \mathbb{R}^{n} \times \mathbb{R}^{m} \times \mathbb{R} & \begin{array}{r}
A u+B x \leq d \\
E u+F x \leq g+\mathbf{1} t \\
c^{T} u \leq \hat{J}^{*}(x)+\varepsilon
\end{array}
\end{array}\right\},
\end{aligned}
$$

where 1 is a vector of ones and $\varepsilon$ is a positive scalar, which is to be solved for all values of $x \in \mathcal{X}_{\varepsilon}$, where

$$
\mathcal{X}_{\varepsilon}:=\widehat{\mathcal{X}} \cap \operatorname{Proj}_{x} \mathcal{Z}_{\varepsilon}
$$

The following theorem provides a means for obtaining $\varepsilon$-optimal solutions to pLPs with strict and non-strict inequality constraints: 
Theorem 2. Consider the optimization problems given in (4), (7) and (8) and let $\varepsilon$ be an arbitrary positive scalar. The following holds:

i) $\mathcal{X}_{\varepsilon}=\widehat{\mathcal{X}}=\operatorname{dom}\left(\hat{J}^{*}\right) \supseteq \mathcal{X}=\operatorname{dom}\left(J^{*}\right)$.

ii) We have that (8) attains its minimum $\forall x \in \mathcal{X}_{\varepsilon}$, and that given any $x \in \mathcal{X}$

$$
\left(u^{*}(x), t^{*}(x)\right) \in \arg \min _{(u, t)}\left\{t \mid(x, u, t) \in \mathcal{Z}_{\varepsilon}\right\} \Rightarrow u^{*}(x) \in \varepsilon-\arg \min _{u \in U(x)} c^{T} u .
$$

iii) The function $V_{\varepsilon}^{*}(\cdot)$ is continuous and piecewise affine on $\mathcal{X}_{\varepsilon}$.

$i v)$ There exists a minimizer function $u^{*}: \mathcal{X}_{\varepsilon} \rightarrow \mathbb{R}^{m}$ that is continuous and PWA.

\section{Proof:}

i) That $\widehat{\mathcal{X}} \supseteq \mathcal{X}$ follows from $\operatorname{Proj}_{x}(\operatorname{cl}(\mathcal{Z})) \supseteq \operatorname{Proj}_{x} \mathcal{Z}$ and

$$
\left\{x \mid \hat{J}^{*}(x)>-\infty\right\}=\left\{x \mid J^{*}(x)>-\infty\right\},
$$

(recall that $J^{*}(x)=\infty$ if $x \notin \mathcal{X}$ ), hence,

$$
\begin{aligned}
& \widehat{\mathcal{X}}=\operatorname{Proj}_{x}(\operatorname{cl}(\mathcal{Z})) \cap\left\{x \mid \hat{J}^{*}(x)>-\infty\right\} \\
& \supseteq \operatorname{Proj}_{x} \mathcal{Z} \cap\left\{x \mid J^{*}(x)>-\infty\right\}=\mathcal{X} .
\end{aligned}
$$

That $\mathcal{X}_{\varepsilon}=\widehat{\mathcal{X}}$ holds trivially by noting that fixing $t=0$ and $\varepsilon=0$ for all $x \in \widehat{\mathcal{X}}$ renders any minimizer $u^{*}(\cdot)$ of (7) feasible also for (8).

ii) Given any $x \in \mathcal{X}_{\varepsilon},(8)$ is an LP and consequently attains its minimum if the infimum is bounded, which is true on $\mathcal{X}_{\varepsilon}$ by construction. Lemma 1 and Assumption 1 ensures that there always exists $\varepsilon$-optimal solutions, i.e.

$$
\forall x \in \mathcal{X} \exists \tilde{u} \in \varepsilon-\arg \min _{u \in U(x)} c^{T} u .
$$

Thus, for any $x \in \mathcal{X}$ and for all $\tilde{u} \in \varepsilon-\arg \min _{u \in U(x)} c^{T} u$ there exists some $\gamma(\tilde{u})<0$ such that

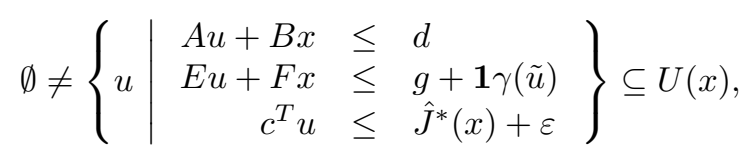

which immediately implies $t^{*}(x)<0$ for all $x \in \mathcal{X}$, and consequently $u^{*}(x) \in$ $\varepsilon-\arg \min _{u \in U(x)} c^{T} u$ for all $x \in \mathcal{X}$, for all

$$
\left(u^{*}(x), t^{*}(x)\right) \in \arg \min _{(u, t)}\left\{t \mid(x, u, t) \in \mathcal{Z}_{\varepsilon}\right\} .
$$

iii) Define a new parameter $y$ and write (8) as

$$
\begin{aligned}
& \bar{V}_{\varepsilon}^{*}(x, y):=\min _{(\bar{u}, \bar{t})}\left\{\bar{t} \mid(x, y, \bar{u}, \bar{t}) \in \overline{\mathcal{Z}}_{\varepsilon}\right\}, \\
& \overline{\mathcal{Z}}_{\varepsilon}:=\left\{\begin{array}{l|l}
(x, y, \bar{u}, \bar{t}) & \begin{array}{r}
A \bar{u}+B x \\
E \bar{u}+F x \\
c^{T} \bar{u} \leq y+\mathbf{1} \bar{t} \\
\leq y+\varepsilon
\end{array}
\end{array}\right\} .
\end{aligned}
$$


Clearly, the above problem is a pLP, and consequently, $\bar{V}_{\varepsilon}^{*}(\cdot, \cdot)$ is continuous and piecewise affine. Defining $y=\hat{J}^{*}(x)$ we see that $\bar{V}_{\varepsilon}^{*}\left(\cdot, \hat{J}^{*}(\cdot)\right)$ is a composition of continuous functions and therefore also a continuous function. Moreover, composition of PWA functions is a PWA function. We clearly also have $V_{\varepsilon}^{*}(x)=\bar{V}_{\varepsilon}^{*}\left(x, \hat{J}^{*}(x)\right)$ for all $x \in \mathcal{X}_{\varepsilon}$.

$i v$ ) Following the same argument as in $i i i)$ we have that there exists a solution $\left(\bar{u}^{*}(\cdot, \cdot), \bar{t}^{*}(\cdot, \cdot)\right)$ to (10) such that $\bar{u}^{*}(\cdot, \cdot)$ is continuous and PWA on its domain, and hence the same holds for $u^{*}(\cdot)=\bar{u}^{*}\left(\cdot, \hat{J}^{*}(\cdot)\right)$.

The pLP is first solved over the closure of $\mathcal{Z}$ to obtain the function $\hat{J}^{*}(\cdot)$. Then solving (8) ensures that we obtain a function $u^{*}(\cdot)$ such that $c^{T} u^{*}(x) \leq J^{*}(x)+\varepsilon$ for all $x \in \mathcal{X}$.

We want to emphasize a key point in the procedure that has to do with the domain of the function $u^{*}(\cdot)$ in the above theorem. The approximate solution has a larger domain than $\mathcal{X}$, since it is solved over the set $\mathcal{Z}_{\varepsilon}$ and not $\mathcal{Z}$. We can restrict any solution (selection) $u^{*}(\cdot)$ to (8) to $\mathcal{X}$ because $\mathcal{X}_{\varepsilon} \supseteq \mathcal{X}$. Thus, in the sequel we let the domain of solutions $u^{*}: \mathcal{X}_{\varepsilon} \rightarrow \mathbb{R}^{m}$ to (8) be restricted to $\mathcal{X}$.

The reason why this restriction is important is because if we want to select the minimum of several affine functions we cannot use the enlarged domains. Comparing Figure 1(b) and 2(a) we see that at $x=5$ the value functions are different. We seek a solution that is within $\varepsilon$ distance of the value function in Figure 1(b), however, if we do not restrict the domain of the approximate solution we would get a solution within $\varepsilon$ distance of the value function in Figure 2(a).

\section{4. $\varepsilon$-optimal solutions for PWA functions}

In the previous section we proposed a procedure for obtaining $\varepsilon$-optimal solutions to pLPs with strict and non-strict inequalities. In this section the procedure is repeatedly applied for the purpose of finding $\varepsilon$-optimal solutions to minimization of PWA functions over FUPs.

Consider the problem of minimizing $f(x, \cdot)$, where $f$ is piecewise affine. We will represent $f$ in the following manner:

$$
f(x, u)=f_{i}(x, u) \quad \text { if } \quad(x, u) \in P_{i} \subseteq \mathbb{R}^{n} \times \mathbb{R}^{m},
$$

where $i \in\{1,2, \ldots, I\}$, each $f_{i}$ is affine and each $P_{i}$ is a polyhedron, thus the domain of $f$ is the FUP $\mathcal{P}:=\operatorname{dom}(f)=\cup_{i=1}^{I} P_{i}$. Note that this implies that for each pair $(i, j) \in\{1,2, \ldots, I\} \times$ $\{1,2, \ldots, I\}$ for which $P_{i} \cap P_{j} \neq \emptyset$ we have $f_{i}(x, u)=f_{j}(x, u), \forall(x, u) \in P_{i} \cap P_{j}$.

Consider the following optimization problem:

$$
J^{*}(x):=\inf _{u}\{f(x, u) \mid(x, u) \in \mathcal{P}\} .
$$

We can clearly represent (11) as

$$
J^{*}(x):=\min _{i \in\{1,2 \ldots, I\}} \inf _{u}\left\{f_{i}(x, u) \mid(x, u) \in P_{i}\right\} .
$$

Observing that $J^{i *}(x):=\inf _{u}\left\{f_{i}(x, u) \mid(x, u) \in P_{i}\right\}$ is a pLP with strict and non-strict inequalities for each $[i \in\{1,2, \ldots, I\}$ we let

$$
\left\{\left(u_{i, \varepsilon}^{*}(\cdot), t_{i, \varepsilon}^{*}(\cdot)\right) \mid i \in\{1,2, \ldots, I\}\right\}
$$


denote a set of continuous selections where each pair $\left(u_{i, \varepsilon}^{*}(\cdot), t_{i, \varepsilon}^{*}(\cdot)\right)$ optimizes the corresponding piecewise pLP defined by (8). Recall also from the previous section that the domain of each $u_{i, \varepsilon}^{*}(\cdot)$ is restricted to the domain of $J^{i *}(\cdot)$, and hence, $\operatorname{dom}\left(f_{i}\left(\cdot, u_{i, \varepsilon}^{*}(\cdot)\right)\right)=\operatorname{dom}\left(J^{i *}\right)$. The following corollary follows from Theorem 2 and the observations above:

Corollary 2. Consider the optimization problem given in (11).

i) For any $\varepsilon>0, x \in \operatorname{dom}\left(J^{*}\right)$ and the problem

$$
J_{\varepsilon}^{*}(x):=\min _{i \in\{1, \ldots, I\}} f_{i}\left(x, u_{i, \varepsilon}^{*}(x)\right),
$$

we have that

$$
\begin{aligned}
j \in \arg & \min _{i \in\{1, \ldots, I\}} f_{i}\left(x, u_{i, \varepsilon}^{*}(x)\right) \\
& \Rightarrow u_{j, \varepsilon}^{*}(x) \in \varepsilon-\arg \min _{u}\{f(x, u) \mid(x, u) \in \mathcal{P}\} .
\end{aligned}
$$

ii) $\operatorname{dom}\left(J_{\varepsilon}^{*}\right)=\operatorname{dom}\left(J^{*}\right)$.

\section{Proof:}

i) Since $\forall x \in \operatorname{dom}\left(J^{i *}\right)$ and $\forall i \in\{1,2, \ldots, I\}$ we have

$$
u_{i, \varepsilon}^{*}(x) \in \varepsilon-\arg \min _{u}\left\{f_{i}(x, u) \mid(x, u) \in P_{i}\right\},
$$

the assertion trivially holds.

ii) This follows by construction; the domain for each $u_{i, \varepsilon}^{*}(\cdot)$ is restricted to the domain of $J^{i *}$, hence the domain of $J_{\varepsilon}^{*}$ is equal to $\cup_{i=1}^{I} \operatorname{dom}\left(J^{i *}\right)$, which is precisely the domain of $J^{*}$.

We revisit Example 1 to illustrate the proposed procedure.

Example 2 (Example 1 cont.) Consider again the problem of minimizing (1a). Although this problem is simple, we formulate the individual pLPs for the purpose of illustrating the procedure. The three pLPs are (where the sets $P_{i}, i=1,2,3$, are defined in Example 1):

$$
\begin{aligned}
& J^{1 *}(x)=\inf _{u}\left\{2 \mid(x, u) \in P_{1}\right\}, \\
& J^{2 *}(x)=\inf _{u}\left\{-u+x \mid(x, u) \in P_{2}\right\}, \\
& J^{3 *}(x)=\inf _{u}\left\{-2 \mid(x, u) \in P_{3}\right\} .
\end{aligned}
$$

Clearly, we only need to use the proposed procedure on the second problem. We solve the pLP over the closure of $P_{2}$ :

$$
\bar{J}^{2 *}(x):=\min _{u}\left\{-u+x \mid(x, u) \in \operatorname{cl}\left(P_{2}\right)\right\} .
$$


Hence, a solution to (14) is $u_{2}^{*}(x)=0$ for all $x \in[-5,5]$, and $\bar{J}^{2 *}(x)=x$. We then continue by finding the $\varepsilon$-optimal solution:

$$
\begin{aligned}
& V_{\varepsilon}^{*}(x)=\min _{(u, t)}\left\{t \mid(x, u, t) \in \mathcal{Z}_{\varepsilon}\right\}, \\
& \mathcal{Z}_{\varepsilon}=\left\{(x, u, t) \mid \begin{array}{rl}
-5 \leq & x \leq 5 \\
u & \leq t \\
-u+x & \leq x+\varepsilon
\end{array}\right\}=\left\{\begin{aligned}
(x, u, t) \mid & x \leq 5 \\
u & \leq t \\
-u & \leq \varepsilon
\end{aligned}\right\},
\end{aligned}
$$

and a solution to (15) is $u^{*}(x)=-\varepsilon$ and hence $f_{2}\left(x, u^{*}(x)\right)=x+\varepsilon$ with $\operatorname{dom}\left(u^{*}\right)=$ $\{x \mid-5 \leq x \leq 5\}$. The final step is to construct $J_{\varepsilon}^{*}(\cdot)$, which clearly becomes

$$
J_{\varepsilon}^{*}(x)=\left\{\begin{array}{llr}
\min \{2, x+\varepsilon\} & \text { if } & -5 \leq x \leq 5 \\
-2 & \text { if } & 5<x \leq 7
\end{array}\right.
$$

and one selection $u_{\varepsilon}^{*}(\cdot)$ that fulfills $f\left(x, u_{\varepsilon}^{*}(x)\right)=J_{\varepsilon}^{*}(x)$ is

$$
u_{\varepsilon}^{*}(x)=\left\{\begin{array}{llr}
-\varepsilon & \text { if } & -5 \leq x<2-\varepsilon \\
0 & \text { if } & 2-\varepsilon \leq x \leq 7
\end{array}\right.
$$

The value function $J_{\varepsilon}^{*}(\cdot)$ is depicted in Figure 3. It is clear that we have obtained a feasible $u_{\varepsilon}^{*}(\cdot)$ such that $f\left(x, u_{\varepsilon}^{*}(x)\right) \leq J^{*}(x)+\varepsilon$ for all $x \in \operatorname{dom}\left(J^{*}\right)$. In addition, it is worth pointing out that by utilizing the procedure of introducing small gaps in the domain of $f$, as described in Example 1, it is not straightforward to compute an error bound, especially if the procedure is repeatedly used in a dynamic programming recursion, as will be done in Section 6.2.

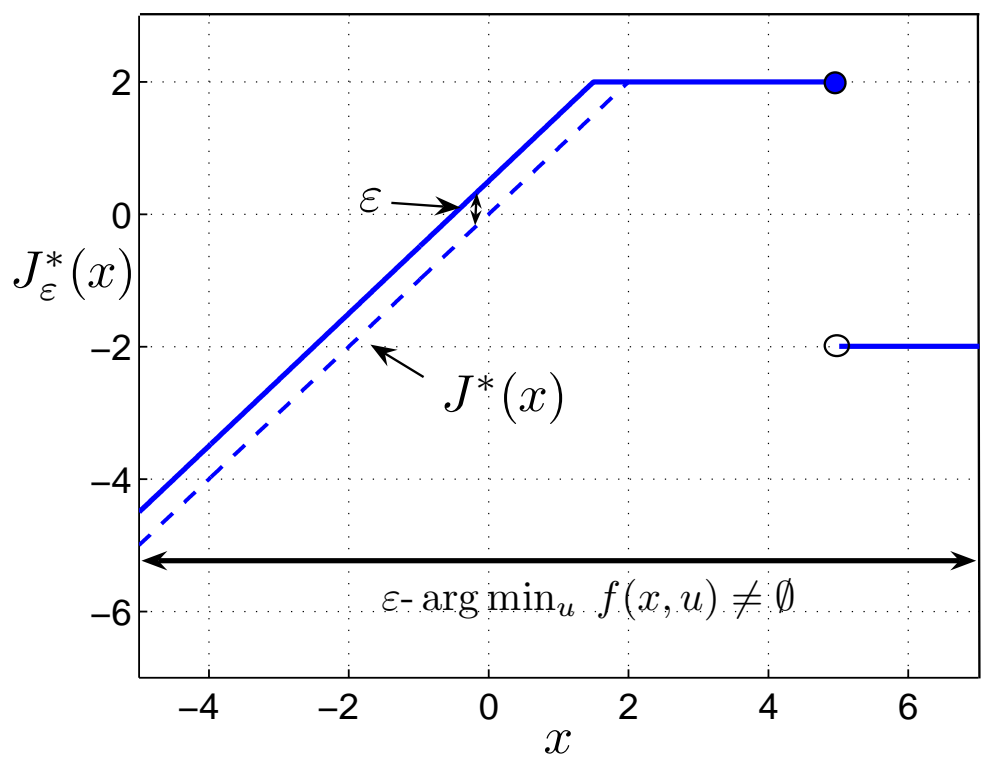

Figure 3. The solid line depicts $J_{\varepsilon}^{*}(\cdot)$. and the dotted line depicts $J^{*}(\cdot)$. Clearly the error is less than $\varepsilon$ everywhere and no gaps are introduced in the domain of the function, cf. Figures 2(a) and 2(b). 
5. $\varepsilon$-optimal solutions to inf-sup of PWA functions

It is apparent from the two preceding sections that a pLP with strict and non-strict inequalities can be viewed as a sub-problem of minimizing a PWA function over a FUP. In this section we extend the approach to inf - sup problems and now minimization of PWA functions become our sub-problems.

Consider the problem

$$
J^{*}(x):=\inf _{u \in \mathcal{U}(x)} \sup _{w \in \mathcal{W}(x, u)} f(x, u, w),
$$

where again we consider the system where $f$ is PWA and defined on the FUP

$$
\mathcal{P}:=\cup_{i=1}^{I} P_{i}, \quad P_{i} \subseteq \mathbb{R}^{n} \times \mathbb{R}^{m} \times \mathbb{R}^{p}, \forall i \in\{1,2 \ldots, I\} .
$$

The $\operatorname{set} \mathcal{U}(\cdot)$ is defined by

$$
\begin{aligned}
\mathcal{Z} & :=\{(x, u) \mid(x, u) \in \mathcal{Y},(x, u, w) \in \Pi \forall w \in \mathcal{W}(x, u)\}, \\
\mathcal{U}(x) & :=\{u \mid(x, u) \in \mathcal{Z}\},
\end{aligned}
$$

where $\mathcal{Y}, \Pi$, and $\operatorname{gph}(\mathcal{W})$ are non-empty FUPs, and $\mathcal{W}(x, u) \neq \emptyset$ for all $(x, u) \in \mathcal{Y}$. The reader is referred to [47] for details on how to compute $\mathcal{Z}$. Define also the sets

$$
\begin{aligned}
\mathcal{X} & :=\operatorname{Proj}_{x} \mathcal{Z}=\{x \mid \exists u \text { s.t. }(x, u) \in \mathcal{Z}\}, \\
\Gamma & :=\{(x, u, w) \mid w \in \mathcal{W}(x, u)\}=\operatorname{gph}(\mathcal{W})=: \cup_{j=1}^{M} \Gamma_{j} .
\end{aligned}
$$

The problem (16) can be divided into a supremum and an infimum problem as

$$
\begin{aligned}
V^{*}(x, u) & :=\sup _{w}\{f(x, u, w) \mid(x, u, w) \in \Gamma\}, \quad \forall(x, u) \in \mathcal{Z}, \\
J^{*}(x) & =\inf _{u}\left\{V^{*}(x, u) \mid(x, u) \in \mathcal{Z}\right\}, \quad \forall x \in \mathcal{X} .
\end{aligned}
$$

Our goal is to obtain a minimizing $u$ with respect to the worst case disturbance $w$. We are not concerned about whether or not there exists $w$ that attains the supremum. Consequently, we do not approximate the supremum problems, but only the infimum problems. Define the FUP

$$
\mathcal{F}:=\cup_{h=1}^{H} F_{h},
$$

where the polyhedra $\left\{F_{1}, F_{2}, \ldots, F_{H}\right\}$ covers the set $\Gamma \cap \mathcal{P}$, and each polyhedron $F_{h}$ is a member of the set

$$
\left\{\Gamma_{j} \cap P_{i} \mid(i, j) \in\{1,2, \ldots, I\} \times\{1,2, \ldots, M\}, \Gamma_{j} \cap P_{i} \neq \emptyset\right\} .
$$

Hence, we can restrict our PWA function $f$ to the domain for which Assumption 1 is valid by defining:

$$
f(x, u . w)=z_{h}(x, u, w) \quad \text { if } \quad(x, u, w) \in F_{h},
$$

where $z_{h}(x, u, w):=f_{i}(x, u, w)$ if $(x, u, w) \in P_{i}$.

For each $h \in\{1,2, \ldots, H\}$ define the pLPs:

$$
\begin{aligned}
\hat{V}_{h}^{*}(x, u) & :=\max _{w}\left\{z_{h}(x, u, w) \mid(x, u, w) \in \operatorname{cl}\left(F_{h}\right)\right\}, \\
V_{h}^{*}(x, u) & :=\sup _{w}\left\{z_{h}(x, u, w) \mid(x, u, w) \in F_{h}\right\} .
\end{aligned}
$$

The following corollary follows from Lemma 2, Corollary 1 and the definitions above: 
Corollary 3. The following holds for all $h \in\{1,2 \ldots, H\}$ :

$$
V_{h}^{*}(x, u)=\hat{V}_{h}^{*}(x, u), \quad \forall(x, u) \in \operatorname{dom}\left(V_{h}^{*}\right) .
$$

Moreover,

$$
V^{*}(x, u)=\left.\max _{h \in\{1,2, \ldots, H\}} \hat{V}_{h}^{*}\right|_{\operatorname{dom}\left(V_{h}^{*}\right)}(x, u) .
$$

Proof: The first assertion holds trivially from the fact that (18a) is a pLP and from the equality of the supremum and maximum over respectively $F_{h}$ and $\mathrm{cl}\left(F_{h}\right)$, cf. Lemma 2 and Corollary 1. Having the first statement established automatically ensures that the second assertion is correct, since, for each $h \in\{1,2, \ldots, H\}$, we restrict $\hat{V}_{h}^{*}(\cdot)$ to the domain of $V_{h}^{*}(\cdot)$.

Since we now have an exact expression for $V^{*}(\cdot)$, we can now apply the procedure from the previous section for the purpose of obtaining an $\varepsilon$-optimal solution to our problem. Recalling that $V^{*}(\cdot)$ is PWA and defined on a FUP $\mathcal{R}=\cup_{k=1}^{K} R_{k}$, that is,

$$
V^{*}(x, u)=V_{k}^{*}(x, u) \quad \text { if } \quad(x, u) \in R_{k},
$$

then $J_{\varepsilon}^{*}(\cdot)$ is defined as

$$
J_{\varepsilon}^{*}(x):=\min _{k \in\{1,2, \ldots, K\}} V_{k}^{*}\left(x, u_{k, \varepsilon}^{*}(x)\right) .
$$

Using Theorem 2 and Corollaries 2 and 3 the following corollary follows easily:

Corollary 4. $J_{\varepsilon}^{*}(x) \leq J^{*}(x)+\varepsilon, \forall x \in \operatorname{dom}\left(J^{*}\right)$, and

$$
\varepsilon-\arg \min _{u}\left\{V^{*}(x, u) \mid(x, u) \in \mathcal{Z}\right\} \neq \emptyset, \quad \forall x \in \operatorname{dom}\left(J^{*}\right) .
$$

Proof: Both statements are confirmed by construction and consequences of Theorem 2 and Corollaries 2 and 3.

6. $\varepsilon$-optimal solutions to inf-sup optimal control of PWA systems

In this section we use the results of the previous sections to obtain $\varepsilon$-optimal solutions to robust optimal control problems for discontinuous PWA systems subject to state- and input-dependent disturbances. We recall the problem setup from [11,12], which was treated in detail in [15] for PWA systems with state- and input-dependent disturbances.

\subsection{Problem setup}

Consider the discrete-time system of the form:

$$
x^{+}=g(x, u, w),
$$

where $x$ is the state, $x^{+}$is the successor state, $u$ is the input, $g(\cdot)$ is assumed piecewise affine on the FUP $\mathcal{P}$, and $w \in \mathcal{W}(x, u) \subseteq \mathbb{R}^{p}$ is a time-varying disturbance. The state and input are subject to 
constraints $(x, u) \in \mathcal{Y} \subseteq \mathbb{R}^{n} \times \mathbb{R}^{m}$, where we assume that $\mathcal{Y}$ is a FUP. The constraints define the set-valued map

$$
\mathcal{U}(x):=\{u \mid(x, u) \in \mathcal{Y}\} .
$$

Let $\pi:=\left\{\mu_{0}(\cdot), \mu_{1}(\cdot), \ldots, \mu_{N-1}(\cdot)\right\}$ denote a control policy (i.e. $\mu_{i}: \mathbb{R}^{n} \rightarrow \mathbb{R}^{m}$ ) over the horizon $N$ and let $\mathbf{w}:=\left\{w_{0}, w_{1}, \ldots, w_{N-1}\right\}$ denote a sequence of disturbances. Moreover, let $\phi(i ; x, \pi, \mathbf{w})$ denote the solution to $x^{+}=g(x, u, w)$ at time-step $i$ for the initial state $x$, control policy $\pi$ and disturbance sequence $\mathbf{w}$.

The cost is defined as

$$
J_{N}(x, \pi, \mathbf{w}):=J_{f}\left(x_{N}\right)+\sum_{i=0}^{N-1} \ell\left(x_{i}, u_{i}\right)
$$

where $x_{i}:=\phi(i ; x, \pi, \mathbf{w})$ and $u_{i}:=\mu_{i}\left(x_{i}\right), \forall i$. The stage cost $\ell(\cdot)$ and terminal cost $J_{f}(\cdot)$ are assumed to be PWA $(p \in\{1, \infty\})$ :

$$
\begin{aligned}
\ell(x, u) & :=\|Q x\|_{p}+\|R u\|_{p}, \\
J_{f}(x) & :=\|P x\|_{p},
\end{aligned}
$$

where $P, Q$, and $R$ are suitably defined weighting matrices.

The optimal control problem considered is given by

$$
\mathbb{P}_{N}(x): J_{N}^{*}(x):=\inf _{\pi \in \Pi_{N}(x)} \sup _{\mathbf{w} \in \mathbf{W}(x, \pi)} J_{N}(x, \pi, \mathbf{w}),
$$

where the set of admissible disturbance sequences is given by

$$
\mathbf{W}(x, \pi):=\left\{\mathbf{w} \mid w_{i} \in \mathcal{W}\left(x_{i}, u_{i}\right), i=0,1, \ldots, N-1\right\},
$$

and the set of admissible control polices is

$$
\Pi_{N}(x):=\left\{\pi \mid\left(x_{i}, u_{i}\right) \in \mathcal{Y}, i=0,1, \ldots, N-1, x_{N} \in X_{f}, \forall \mathbf{w} \in \mathbf{W}(x, \pi)\right\},
$$

where $X_{f}$ the target set which is a non-empty FUP. In the sequel, we denote by $X_{N}$ the set of initial states for which there exists an admissible control policy, i.e.

$$
X_{N}:=\left\{x \mid \Pi_{N}(x) \neq \emptyset\right\} .
$$

In addition we make the following assumptions in order to ensure that $\mathbb{P}_{N}(x)$ fits into the dynamic programming procedure from $[11,15]$ and that each sub-problem in the recursion reduces to optimization of a PWA function over a FUP:

A1: The function $g: \mathcal{P} \rightarrow \mathbb{R}^{n}$ is PWA on the FUP $\mathcal{P}$.

A2: The sets $\mathcal{Y}$ and $X_{f}$ are non-empty FUPs.

A3: For all $(x, u) \in \mathcal{Y}$, the set $\mathcal{W}(x, u)$ is non-empty.

A4: The graph of $\mathcal{W}$ is a non-empty FUP.

A5: The value function $J_{N}^{*}(\cdot)$ is bounded above and below on $X_{N}$, and $X_{N}$ is non-empty.

Thus, in comparison to [15] several assumptions are relaxed (note that we use the definition in [39] for continuity of a set-valued map):

i) We do not assume that $g(\cdot)$ is continuous. 
ii) $\mathcal{Y}$ and $X_{f}$ are not required to have the origin in the interior.

iii) The set-valued map $x \mapsto \mathcal{U}(x)$ is not required to be continuous and bounded on bounded sets.

$i v)$ The set-valued map $(x, u) \mapsto \mathcal{W}(x, u)$ is not required to be continuous.

$v)$ The solution to $\mathbb{P}_{N}(x)$ is not assumed to exist $\forall x \in X_{N}$.

It should be noted that in [15] the majority of the assumptions above are made for the purpose of being able to directly apply the topological results in [47].

\subsection{Sub-optimal solution via dynamic programming.}

The procedure for obtaining approximate solutions to (21), under assumption A1-A5, will be presented in this section. The results in [47] reveal geometric structure that lead to the possibility to apply a dynamic programming approach [9-11] to the above mentioned problem. We recall the dynamic programming method from [15], which is a specialization of the method in [11] to PWA systems.

Dynamic programming provides a recursive procedure for computing sequentially the partial return functions $J_{j}^{*}(\cdot)$ (defined in (21) with $N=j$ ), the associated set-valued control laws $\kappa_{j}(\cdot)$ as well as their domains (here $j$ denotes 'time-to-go' so that $\kappa_{j}(\cdot)=\mu_{N-j}^{*}(\cdot)$ if $j \in\{1, \ldots, N\}$ where $\mu_{j}(\cdot)$ is as defined in the previous section). The domain of $J_{j}^{*}(\cdot)$ and $\kappa_{j}(\cdot)$ is $X_{j}$, the set of states that can be robustly steered to the terminal set $X_{f}$ in $j$ steps or less. Define also

$$
g(x, u, \mathcal{W}(x, u)):=\{g(x, u, w) \mid w \in \mathcal{W}(x, u)\} .
$$

The recursion for obtaining a solution to $\mathbb{P}_{N}(x)$ can be found in $[11,15]$, and is as follows: For all $j \in\{1,2, \ldots\}, j$ denotes "time-to-go", and the partial return function $J_{j}^{*}(\cdot)$, the set-valued control law $\kappa_{j}(\cdot)$, and the controllability set $X_{j}$ are given by:

$$
\begin{aligned}
J_{j}^{*}(x) & =\inf _{u \in \mathcal{U}(x)} \sup _{w \in \mathcal{W}(x, u)}\left\{\tilde{J}_{j-1}(x, u, w) \mid g(x, u, \mathcal{W}(x, u)) \subseteq X_{j-1}\right\}, \forall x \in X_{j}, \\
\tilde{J}_{j-1} & :=\ell(x, u)+J_{j-1}^{*}(g(x, u, w)), \\
\kappa_{j}(x) & =\underset{u \in \mathcal{U}(x)}{\arg \min }\left\{\ell(x, u)+J_{j-1}^{*}\left(x^{+}\right) \mid g(x, u, \mathcal{W}(x, u)) \subseteq X_{j-1}\right\}, \\
X_{j} & =\left\{x \mid \exists u \in \mathcal{U}(x) \text { s.t. } g(x, u, \mathcal{W}(x, u)) \subseteq X_{j-1}\right\},
\end{aligned}
$$

with boundary conditions

$$
J_{0}^{*}(x)=J_{f}(x), \quad X_{0}=X_{f} .
$$

The conditions $g(x, u, \mathcal{W}(x, u)) \subseteq X_{j-1}$ and $u \in \mathcal{U}(x)$ in (22) may be expressed as

$$
(x, u) \in \Sigma_{j}:=\left\{(x, u) \in \mathcal{Y} \mid g(x, u, w) \in X_{j-1} \quad \forall w \in \mathcal{W}(x, u)\right\},
$$

in which case $X_{j}$ can be interpreted as the projection of the set $\Sigma_{j}$, i.e.

$$
X_{j}=\left\{x \mid \exists u \text { s.t. }(x, u) \in \Sigma_{j}\right\} .
$$

The reader is referred to [47] for details on how to compute $\Sigma_{j}$. In order to analyze $\mathbb{P}_{N}(x)$, it is convenient to introduce the functions $V_{j}^{*}(\cdot), j=1,2, \ldots, N-1$, defined by

$$
V_{j}^{*}(x, u):=\sup _{w \in \mathcal{W}(x, u)} J_{j}^{*}(g(x, u, w)) .
$$


Note that we are interested in values of the functions $\left\{V_{j}^{*}(\cdot)\right\}_{j=1}^{N-1}$ and the sets $\left\{\Sigma_{j}\right\}_{j=1}^{N-1}$. The recursion (22a)-(22d) may therefore be rewritten as

$$
\begin{aligned}
V_{j-1}^{*}(x, u) & =\sup _{w}\left\{J_{j-1}^{*}(g(x, u, w)) \mid w \in \mathcal{W}(x, u)\right\} \quad \forall(x, u) \in \Sigma_{j}, \\
J_{j}^{*}(x) & =\inf _{u}\left\{\ell(x, u)+V_{j-1}^{*}(x, u) \mid(x, u) \in \Sigma_{j}\right\} \quad \forall x \in X_{j}, \\
\kappa_{j}(x) & =\underset{u}{\arg \min }\left\{\ell(x, u)+V_{j-1}^{*}(x, u) \mid(x, u) \in \Sigma_{j}\right\} \quad \forall x \in X_{j}, \\
\Sigma_{j} & =\left\{(x, u) \in \mathcal{Y} \mid g(x, u, \mathcal{W}(x, u)) \subseteq X_{j-1}\right\}, \\
X_{j} & =\left\{x \mid \exists u \text { s.t. }(x, u) \in \Sigma_{j}\right\} .
\end{aligned}
$$

For each $j$ we propose to use the approximate procedure presented in the previous sections (Theorems 2-3) when solving (23b) when the set $\kappa_{j}(\cdot)$ (23c) is empty. Thus, for each $j$ for which $\kappa_{j}(x)=\emptyset$ for some $x \in X_{j}$, we compute the approximation $\kappa_{j, \varepsilon}(\cdot)$, that is, $\kappa_{j, \varepsilon}(\cdot)$ is a selection from the set-valued map $x \mapsto \varepsilon-\arg \min _{u}\left\{\ell(x, u)+V_{j-1}^{*}(x, u) \mid(x, u) \in \Sigma_{j}\right\}$.

Two approaches are natural when considering the dynamic programming recursion; the first is the one outlined above, namely using the exact expressions for the functions $\left\{V_{j}^{*}(\cdot)\right\}_{j=0}^{N-1}$ and $\left\{J_{j}^{*}(\cdot)\right\}_{j=0}^{N}$ and use Theorem 2 and Corollaries 2-3 to compute $\left\{\kappa_{j, \varepsilon}(\cdot)\right\}_{j=1}^{N}$. The second approach is to use the approximate value function in the dynamic programming recursion:

$$
\begin{aligned}
V_{j-1, \varepsilon}^{*}(x, u) & =\sup _{w}\left\{J_{j-1, \varepsilon}^{*}(g(x, u, w)) \mid w \in \mathcal{W}(x, u)\right\}, \quad \forall(x, u) \in \Sigma_{j}, \\
\kappa_{j, \varepsilon}(x) & \in \varepsilon-\arg \min _{u}\left\{\ell(x, u)+V_{j-1, \varepsilon}^{*}(x, u) \mid(x, u) \in \Sigma_{j}\right\}, \quad \forall x \in X_{j}, \\
J_{j, \varepsilon}^{*}(x) & =\ell\left(x, \kappa_{j, \varepsilon}(x)\right)+V_{j-1, \varepsilon}^{*}\left(x, \kappa_{j, \varepsilon}(x)\right), \quad \forall x \in X_{j} .
\end{aligned}
$$

A sketch of the final procedure is given in Procedure 1.

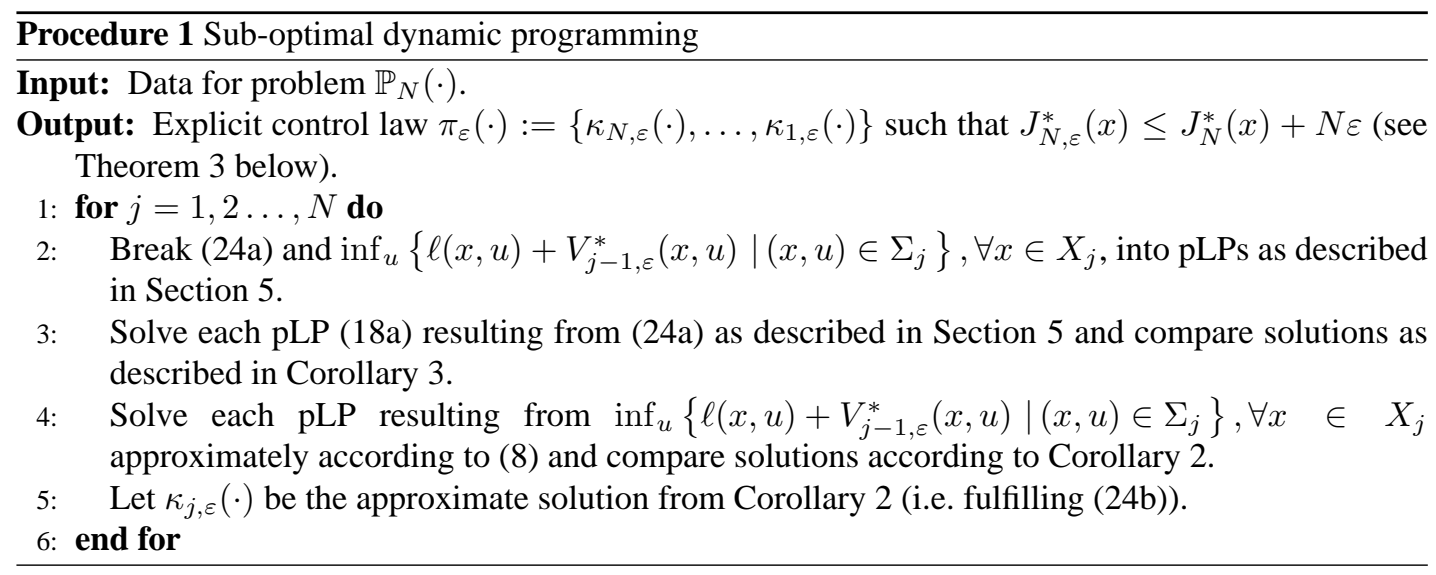

With this approach an error bound is easily derived, as demonstrated by the following theorem:

Theorem 3. $J_{N, \varepsilon}^{*}(x) \leq J_{N}^{*}(x)+N \varepsilon$.

\section{Proof:}


We verify the induction base by carrying out the first iteration of the dynamic programming recursion:

$$
\begin{aligned}
V_{0}^{*}(x, u) & =\sup _{w}\left\{J_{0}^{*}(g(x, u, w)) \mid w \in \mathcal{W}(x, u)\right\}, \quad \forall(x, u) \in \Sigma_{1}, \\
\kappa_{1, \varepsilon}(x) & \in \varepsilon-\arg \min _{u}\left\{\ell(x, u)+V_{0}^{*}(x, u) \mid(x, u) \in \Sigma_{1}\right\}, \quad \forall x \in X_{1}, \\
J_{1, \varepsilon}^{*}(x) & =\ell\left(x, \kappa_{1, \varepsilon}(x)\right)+V_{0}^{*}\left(x, \kappa_{1, \varepsilon}(x)\right) \leq J_{1}^{*}(x)+\varepsilon .
\end{aligned}
$$

Assuming that the bound holds for $N=j$, i.e.

$$
J_{j, \varepsilon}^{*}(x) \leq J_{j}^{*}(x)+j \varepsilon
$$

we must verify that the bound also holds for $N=j+1$. We get:

$$
\begin{aligned}
V_{j, \varepsilon}^{*}(x, u) & =\sup _{w}\left\{J_{j, \varepsilon}^{*}(g(x, u, w)) \mid w \in \mathcal{W}(x, u)\right\} \\
& \leq \sup _{w}\left\{J_{j}^{*}(g(x, u, w)) \mid w \in \mathcal{W}(x, u)\right\}+j \varepsilon \\
& =V_{j}^{*}(x, u)+j \varepsilon, \quad \forall(x, u) \in \Sigma_{j+1},
\end{aligned}
$$

and

$$
\kappa_{j+1, \varepsilon}(x) \in \varepsilon-\arg \min _{u}\left\{\ell(x, u)+V_{j, \varepsilon}^{*}(x, u) \mid(x, u) \in \Sigma_{j+1}\right\},
$$

and since

$$
\begin{aligned}
\inf _{u}\{\ell(x, u) & \left.+J_{j, \varepsilon}^{*}(x, u) \mid(x, u) \in \Sigma_{j+1}\right\} \\
\leq & \overbrace{\inf _{u}\left\{\ell(x, u)+V_{j}^{*}(x, u) \mid(x, u) \in \Sigma_{j+1}\right\}}^{J_{j+1}^{*}(x)}+j \varepsilon
\end{aligned}
$$

we get

$$
J_{j+1, \varepsilon}^{*}(x)=\ell\left(x, \kappa_{j+1, \varepsilon}(x)\right)+V_{j, \varepsilon}^{*}\left(x, \kappa_{j+1, \varepsilon}(x)\right) \leq J_{j+1}^{*}(x)+(j+1) \varepsilon .
$$

With this approach it is clear that the degree of sub-optimality can be specified a priori by choosing $\varepsilon$.

Finally, we would like to point out that stability of PWA systems with sub-optimal control laws has been studied in the control literature. The reader is referred to [48] for input-to-state stability analysis for PWA systems under sub-optimal control laws.

\section{Conclusion}

A method for obtaining approximate solutions to robust optimal control of discontinuous PWA systems has been presented. This was achieved by repeatedly applying a procedure, which obtained $\varepsilon$-optimal solutions to pLPs with strict and non-strict inequality constraints, in a dynamic programming approach. It has been demonstrated that $\varepsilon$-optimal solutions exists under mild assumptions, a bound on the total error for the approximate dynamic programming has been given and the degree of sub-optimality can be specified a priori.

This chapter considered the problem of computing the solution to a given finite horizon optimal control problem. Though this is an interesting and practically useful problem in itself, an interesting research question is how to modify the problem so that stability may be guaranteed if the solution were to be implemented in a receding horizon fashion, as common in MPC [4]. 


\section{Acknowledgements}

This research is part of the Strategic University Program on Computational Methods for Nonlinear Motion Control funded by the Research Council of Norway. The second author is a Royal Academy of Engineering Research Fellow and would like to thank the Royal Academy of Engineering, UK, for their support.

\section{REFERENCES}

1. T. Gal and J. Nedoma. Multiparametric linear programming. Management Science, 18:406-442, 1972.

2. B. Bank, J. Guddat, D. Klatte, B. Kummer, and K. Tammer. Non-linear Parametric Optimization. Birkhäuser, Berlin, 1983.

3. M. Schechter. Polyhedral functions and multiparametric linear programming. Journal of Optimization Theory and Applications, 53:269-280, 1987.

4. D. Q. Mayne, J. B. Rawlings, C. V. Rao, and P. O. M. Scokaert. Constrained model predictive control: Stability and optimality. Automatica, 36:789-814, 2000.

5. A. Bemporad, M. Morari, V. Dua, and E. N. Pistikopoulos. The explicit linear quadratic regulator for constrained systems. Automatica, 38:3-20, 2002.

6. A. Bemporad, F. Borrelli, and M. Morari. Model predictive control based on linear programming - The explicit solution. IEEE Trans. on Automatic Control, 47:1974-1985, 2002.

7. M. M. Seron, G. C. Goodwin, and J. A. De Doná. Characterisation of receding horizon control for constrained linear systems. Asian Journal of Control, 5:271-286, 2003.

8. R. E. Bellman. Dynamic Programming. Princeton University Press, Princeton, NJ, 1957.

9. H. Witsenhausen. A minimax control problem for sampled linear systems. IEEE Transactions on Automatic Control, 13:5-21, 1968.

10. D. P. Bertsekas and I. B. Rhodes. Sufficiently informative functions and the minimax feedback control of uncertain dynamic systems. IEEE Transactions on Automatic Control, 18:117-124, 1973.

11. D. P. Bertsekas. Dynamic programming and optimal control, volume 1. Athena Scientific, Belmont, MA, 2nd edition, 2000.

12. E. C. Kerrigan and D. Q. Mayne. Optimal control of constrained, piecewise affine systems with bounded disturbances. In Proc. 41st IEEE Conf. on Decision and Control, pages 1552-1557, Las Vegas, NV, 2002.

13. D. M. de la Peña, T. Alamo, A. Bemporad, and E. F. Camacho. A dynamic programming approach for determining the explicit solution of MPC controllers. In Proc. 43rd IEEE Conference on Decision and Control, Paradise Island, Bahamas, 2002.

14. M. Diehl and J. Björnberg. Robust dynamic programming for min-max model predictive control of constrained uncertain systems. IEEE Trans. Automatic Control, 49:2253-2257, 2004

15. S. V. Raković, E. C. Kerrigan, and D. Q. Mayne. Optimal control of constrained piecewise affine systems with state- and input-dependent disturbances. In Proc. 16th International Symposium on Mathematical Theory of Networks and Systems, Katholieke Universiteit Leuven, Belgium, 2004.

16. F. Borrelli, M. Baotić, A. Bemporad, and M. Morari. Dynamic programming for constrained optimal control of discretetime linear hybrid systems. Automatica, 41:1709-1721, 2005.

17. D. Q. Mayne, S. V. Rakovć, R. B. Vinter, and E. C. Kerrigan. Characterization of the solution to a constrained $H_{\infty}$ optimal control problem. Automatica, 42:371-382, 2006.

18. B. Lincoln and A. Rantzer. Relaxing dynamic programming. IEEE Transactions on Automatic Control, 51:1249-1260, 2006.

19. A. Rantzer. Relaxed dynamic programming in switching systems. Proc. IEE Control Theory and Applications, 153:567574, 2006.

20. D. Q. Mayne and S. V. Raković. Optimal control of constrained piecewise affine systems using reverse transformation. In Proc. 41st IEEE Conference on Decision and Control, pages 1546-1551, Las Vegas, USA, 2002.

21. A. Bemporad, F. Borrelli, and M. Morari. Piecewise linear optimal controllers for hybrid systems. In Proc. American Contr. Conf., pages 1190-1194, Chicago, IL, 2000.

22. A. Bemporad, F. Borrelli, and M. Morari. Optimal Controllers for Hybrid Systems: Stability and Piecewise Linear Explicit Form. In Proc. 39th IEEE Conf. on Decision and Control, pages 1810-815, Sydney, 2000.

23. F. Borrelli. Discrete Time Constrained Optimal Control. PhD thesis, Swiss Federal Institute of Technology, Zurich, Switzerland, 2002.

24. Ph. Rostalski, Th. Besselmann, M. Barić, and M. Morari. A Hybrid Approach to Modelling, Control and State estimation of Mechanical Systems with Backlash. International Journal of Control, 80:1729-1740, 2007. 
25. W. P. M. H. Heemels, B. De Shutter, and A. Bemporad. Equivalence of hybrid dynamical models. Automatica, 37:10851091, 2001

26. T. A. Johansen. On multi-parametric nonlinear programming and explicit nonlinear model predictive control. In Proc. 41st IEEE Conf. on Decision and Control, pages 2768-2773, Las Vegas, NV, 2002.

27. T. A. Johansen. Approximate explicit receding horizon control of constrained nonlinear systems. Automatica, 40:293-300, 2004.

28. A. Grancharova and T. A. Johansen. Explicit min-max model predictive control of constrained nonlinear systems with model uncertainty. In Porc. 16th IFAC World Congress, Prague, Czech Republic, 2005.

29. A. Bemporad and C. Filippi. An algorithm for approximate multiparametric convex programming. Computational Optimization and Applications, 35:87-108, 2006.

30. C. N. Jones, E. C. Kerrigan, and J. M. Maciejowski. Lexicographic perturbation for multiparametric linear programming with applications to control. Automatica, 43:1808-1816, 2007.

31. J. Spjøtvold, P. Tøndel, and T. A. Johansen. A method for obtaining continuous solutions to multiparametric linear programs. In Proc. IFAC World Congress on Automatic Control, Prague, 2005.

32. C.N. Jones and Jan M. Maciejowski. Reverse Search for Parametric Linear Programming. In Proc. 45th IEEE Conf. on Decision and Control, pages 1504-1509, San Diego, CA, 2006.

33. F. Borrelli, A. Bemporad, and M. Morari. A geometric algorithm for multi-parametric linear programming. Journal of Optimization Theory and Applications, 118:515-540, 2003.

34. F. J. Christophersen, M. Kvasnica, C. N. Jones, and M. Morari. Efficient evaluation of piecewise control laws defined over a large number of polyhedra. In Proc. of the European Control Conference, Kos, Greece, 2007.

35. C. N. Jones, P. Grieder, and S. V. Raković. A logarithmic-time solution to the point location problem for parametric linear programming. Automatica, 42:2215-2218, 2006

36. P. Tøndel, T. A. Johansen, and A. Bemporad. Computation of piecewise affine control via binary search tree. Automatica, 39:945-950, 2003

37. A. Bemporad and M. Morari. Control of systems integrating logic, dynamics, and constraints. Automatica, 35:407-427, 1999.

38. R. Goebel and A. R. Teel. Solutions to hybrid inclusions via set and graphical convergence with stability theory applications. Automatica, 42:573-587, 2006.

39. R. T. Rockafellar and R. J-B. Wets. Variational Analysis, volume 317 of Grundlehren der mathematischen Wissenschaften. Springer-Verlag, Berlin, 1998.

40. G. B. Dantzig, J. Folkman, and N. Z. Shapiro. On the continuity of the minimum set of a continuous function. Journal of Mathematical Analysis and Applications, 17:519-548, 1967.

41. C. Berge. Topological Spaces. Oliver and Boyd Ltd, London, 1963.

42. F. Hausdorff. Set Theory. Chelsea Publishing Company, New York, 1957.

43. J. P. Aubin and Hélène Frankowska. Set-Valued Analysis. Birkhäuser, Boston, 1990.

44. J. Spjøtvold, P. Tøndel, and T. A. Johansen. A continuous selection and unique polyhedral representation of solutions to convex parametric quadratic programs. Journal of Optimization Theory and Applications, 133:177-189, 2007.

45. C. N. Jones. Polyhedral Tools for Control. PhD thesis, Cambridge University, Cambridge, UK, 2005.

46. R. T. Rockafellar. Convex Analysis. Princeton Univeristy Press, Princeton, New Jersey, 1972.

47. S. V. Raković, E. C. Kerrigan, D. Q. Mayne, and J. Lygeros. Reachability analysis of discrete-time systems with disturbances. IEEE Transactions on Automatic Control, 51:546-561, 2006.

48. M. Lazar and W.P.M.H. Heemels (2008). Predictive control of hybrid systems: Input-to-state stability results for suboptimal solutions. Automatica, 2008. in press. 